\title{
Water Wave Optimization Algorithm based Congestion Control and Quality of Service Improvement in Wireless Sensor Networks
}

\author{
Mukhdeep Singh Manshahia \\ Punjabi University, Patiala \\ mukhdeep@gmail.com
}

\begin{abstract}
Many researchers have implemented various machine learning algorithms and verify their results with the existing algorithms to control congestion in Wireless Sensor Networks. The major challenge lies in developing an algorithm which optimizes the value of the objective function on the basis of parameters like network throughput, residual energy and packet loss rate of the nodes in the network. An objective function based on these parameters is proposed in the present work. Water wave optimization algorithm is applied on the objective function and an optimum solution is obtained. The proposed approach is compared with the Congestion Detection and Avoidance algorithm (CODA) and Particle Swarm Optimization Algorithm (PSO). The proposed solution outperforms both algorithms on the basis of various performance parameters.
\end{abstract}

Keywords: Water wave optimization algorithm, Congestion control, Wireless Sensor Networks

\section{Introduction}

Wireless sensor networks (WSNs) are the sensors deployed in physical environment which are controlled by a central receiving unit called Base Station. Base Station is responsible for the collection and processing of data collected from various different types of sensors [1, 2]. The sensors deployed in the network are either of the same type or of different types depending on the application [3]. Open-air environments need many sensor nodes to cover a large area whereas fewer sensor nodes are needed in indoor environments in a sensor network [4, 5]. High number sensors are deployed in the industrial environment to enhance coverage, fetch live data and take precise decisions [6]. Lots of potential applications of wireless sensor networks make wireless sensor networks a fast growing market $[7,8]$.

Network lifespan and congestion are two basic performance issues in sensing networks. The energy of the wireless sensor nodes is spent during transmission of data from sensor to sink, sensing the particular environment and processing of data [9]. Congestion can occur while transmitting the data from sensor to sink node. Network congestion can shortened the lifetime of sensor nodes due to packet loss and retransmissions. Therefore minimizing congestion and the energy consumption is the key demands among the sensor network protocols and algorithms. 
Mukhdeep Manshahia; Water Wave Optimization Algorithm based Congestion Control and Quality of Service Improvement in Wireless Sensor Networks, Transactions on Networks and Communications, Volume 5 No. 4, August (2017); pp: 31-39

\section{Literature Survey}

Motdhare et al. [10] provided a congestion control mechanism for WSN using a mobile sink to avoid congestion and lifetime maximization. They have analyzed the impact of mobile sink in congestion reduction and increasing lifetime of the sensor network by considering various parameters and compared it with static sink. Simulation results have shown that proposed technique is effective for congestion avoidance and better lifetime of WSN.

Antoniou et al. [11] proposed swarm Intelligence inspired congestion control mechanism for WSNs. They have provided congestion avoidance and control mechanism by mimicking the obstacle avoidance behavior of bird flock. Packets are considered as flocks who are trying to avoid the congested regions while moving towards sink. At each node, a packet interacts with the neighboring nodes and each packet got attracted magnetically towards sink. Evaluation results have shown that proposed approach perform better in load balancing, robustness against failing nodes and scalability.

Luha et al. [12] proposed Redundancy Aware Hierarchical Tree Alternative Path (RAHTAP) algorithm to control congestion in WSNs. RAHTAP have managed to control congestion in WSNs using redundancy detection and elimination whenever congestion occurs in the network. Elimination of the duplicate packets at each sensor nodes has increased throughput and efficiency. Simulation results have shown that the proposed method is better in confronting over-burden circumstances in Wireless Sensor Networks.

Wei-qiang et al. [13] proposed penalty function-based optimal congestion control (POCC) algorithm with the notion of link's interference set. Each link transmits the information on congestion state to its interference set periodically. Simulation results have proved that POCC is effective in efficient and fair resource allocation.

Raghunathan et al. [14] presented that the results related to stability and fairness of TCP-AIMD cannot be used in wireless networks due to broadcast nature of wireless networks. Wireless congestion control is complicated issue because of interference like self-interference and inters flow interference. In wireless channel, Packet transmission by neighbouring nodes may not be simultaneously due to interference. Ns2 based simulation results have indicated oscillatory behaviour.

Zhao et al. [15] proposed energy effective congestion control for wireless ad hoc network. They have used source node rate control to control congestion and intermediate node power allocation to eliminate the bottleneck. Dual decomposition has been proposed to decompose the problem into intermediate node power allocation and source flow control. Numerical simulation results have shown that the proposed approach increases network performance and reduces the energy consumption of nodes.

\section{Problem Formulation}

A fitness function is considered based on various parameters like network throughput, residual energy and packet loss rate to control congestion in WSN $[16,17]$ :

Fitness Function $\left(f_{j}\right)=\sum_{i=1}^{N}\left(w_{1} * \tau_{i}+w_{2} *\left(1-P_{L_{i}}\right)+w_{3} * E_{i}+w_{4} * d_{j, i}\right)$

Where, $i$ is the iteration which ranges from 1 to $\mathrm{N}$ (total number of nodes),

$w_{1}, w_{2}, w_{3}$ and $w_{4}$ are the weights supplied to the algorithm, 
$\tau$ is the throughput of the network,

$P_{L}$ is the normalized Packet lost rate in the network

$d_{j, i}$ is the distance between node $i$ and $j$ and

$E$ is the residual energy of each node in the network.

\section{Proposed Methodology}

In the proposed approach, Water wave optimization algorithm is implemented to solve congestion problem in WSN. Water Wave Optimization algorithm is based on the shallow water wave models for solving optimization problems [18]. It is studied as when a wave travels from deep water to shallow water, its wave height increases and its wavelength decreases and vice versa. Without losing generality, suppose we have a maximization problem with objective function $\mathrm{f}$. In Water Wave Optimization, the solution space $\mathrm{X}$ is analogous to the seabed area, and the fitness of a point $\mathrm{x} \in \mathrm{X}$ is measured inversely by its seabed depth: the shorter the distance to the still water level, the higher will be the fitness $\mathrm{f}(\mathrm{x})$. During the problem-solving process, we consider three types of operations on the waves: Propagation, Refraction, and Breaking [18].

\subsection{The Water Wave Algorithm}

1 Randomly initializes a population P of $n$ waves (solutions);

2 while stop criterion is not satisfied do

3 for each $x \in P$ do

$4 \quad$ Propagate $x$ to a new $x^{\prime}$;

$5 \quad$ if $f\left(x^{\prime}\right)>f(x)$ then

6

if $f\left(x^{\prime}\right)>f\left(x^{*}\right)$ then

7

Break $x^{\prime}$;

8

Update $x^{*}$ with $x^{\prime}$;

9

Replace $x$ with $x^{\prime}$;

10

else

Decrease X.h by one;

if $x . h>0$ then

Refract $x$ to a new $x^{\prime}$;

14 Update the wavelengths;

\section{5 return $x^{*}$}

Water wave algorithm is applied to detect and reduce congestion in WSN. The objective is to maximize the throughput of network by optimizing the objective function.

Three types of operations are performed on a wave: Propagation, Refraction and Breaking. In deep water, the depth of the sea or the distance of the seabed from the surface is considered as the residual energy of the node. 
Mukhdeep Manshahia; Water Wave Optimization Algorithm based Congestion Control and Quality of Service Improvement in Wireless Sensor Networks, Transactions on Networks and Communications, Volume 5 No. 4, August (2017); pp: 31-39

This also represents the nodes which are less used or which have larger energy as compared to every other node in the network. So, our route consists of those nodes. The congestion will be high near the high wave i.e. around shallow water and these nodes are considered as congested nodes. So the packet loss rate will be high in these nodes. By putting these values in the objective function, we have tried to find the optimal value of the route and thus try to maximize the throughput of the network.

Figure 2 shows the flow diagram of the proposed approach of using the refraction, breaking and propagation techniques for congestion control in wireless sensor networks.

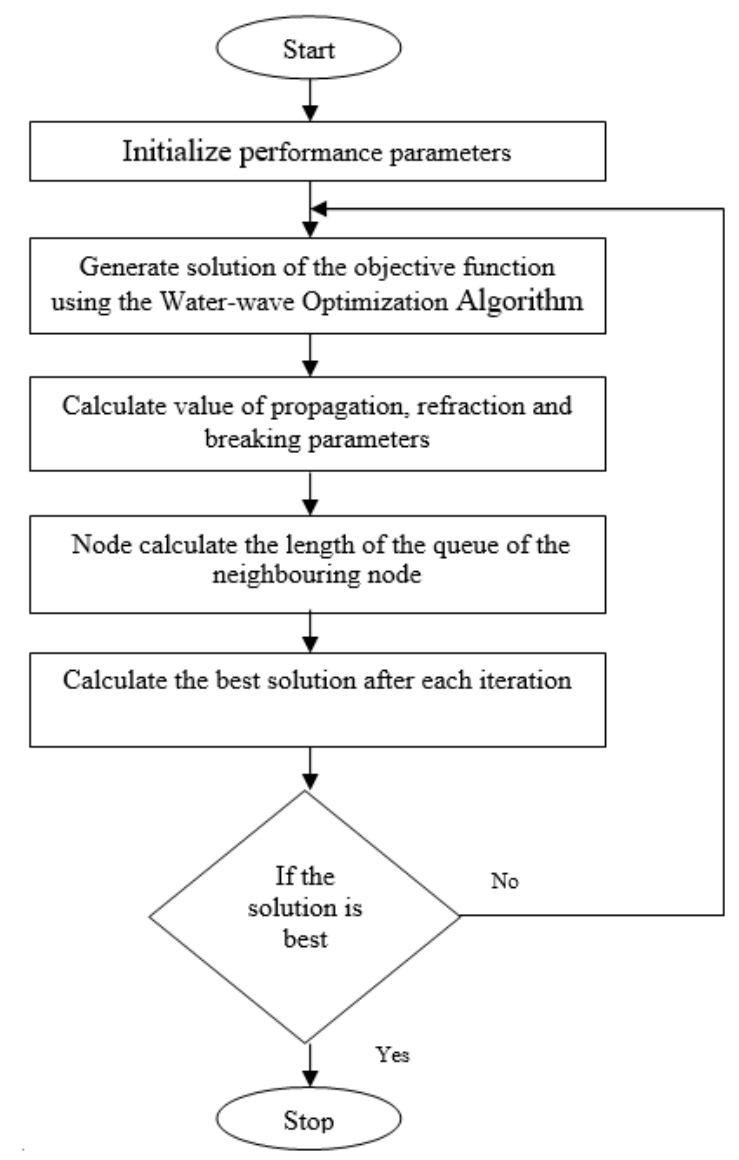

Figure. 2 Flow Diagram of the Proposed Methodology

We are comparing our proposed approach with Congestion Detection and Avoidance (CODA) [19] and particle swarm optimization (PSO) based congestion control approach [20, 21, 22].

\section{Simulation Environment}

We have placed 50 nodes in a 1000*1000 size grid using Network Simulator (NS)-2.35. Each node in the network is configured to wireless channel and Omni-directional antenna type. MAC layer is used with standard IEEE 802.11 and the radio model has been adopted $[23,24,25]$. Table 1 shows the simulation parameters used in the simulation. 
Table 1: Simulation Parameters

\begin{tabular}{|c|c|}
\hline Parameter & Value \\
\hline No. of Nodes & 50 \\
\hline X dimension & 1000 \\
\hline Y dimension & 1000 \\
\hline Grid Area & $1000 * 1000$ \\
\hline Mac Protocol & IEEE 802.11 \\
\hline Propagation Model & Two-Ray Ground model \\
\hline Transmission Range & 200m (approx.) \\
\hline Source Traffic & Omni-Antenna \\
\hline Antenna type & Wireless Channel \\
\hline Channel type & AODV \\
\hline Routing Protocol & 60 \\
\hline Simulation time & Prequeue \\
\hline Interface Queue type &
\end{tabular}

\section{Results And Discussions}

Queue Length of each node in the network is inversely proportional to the number of hops in the network and with increase in number of hops, queue length decreases as shown in figure 3 . The packets flowing in the network can adapt alternate routes and thereby decreases the congestion in the network.

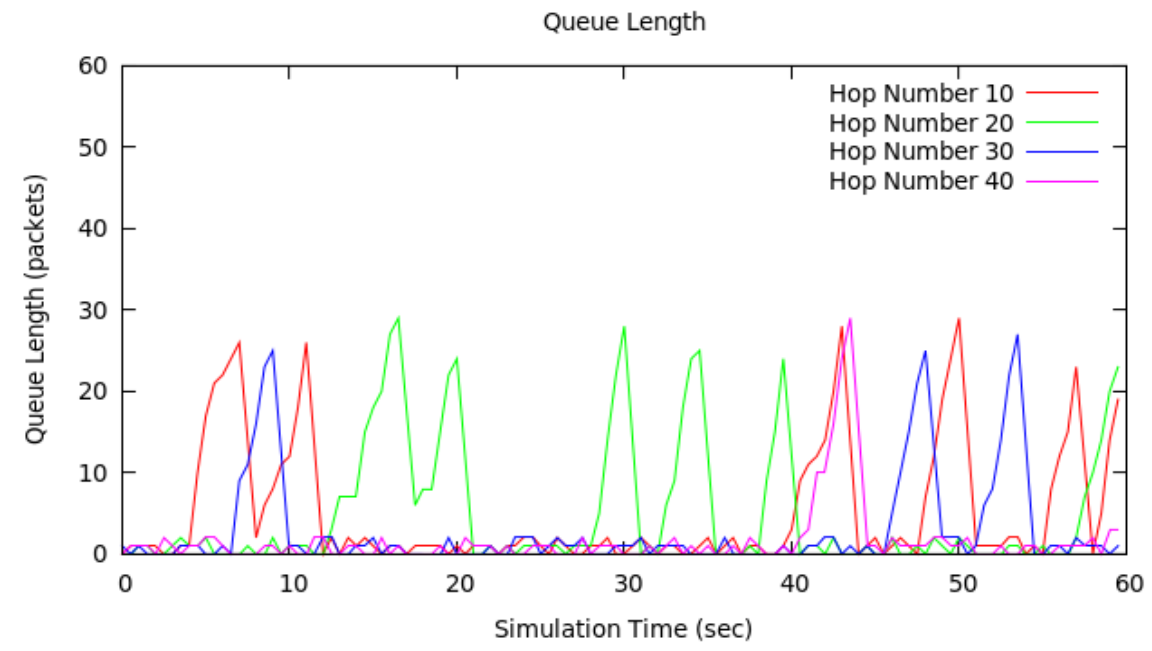

Figure. 3 Graph between Simulation Time and Queue Length for Hop count 10, 20, 30 and 40.

Figure 4 shows that the value of fitness function decreases with increase in number of hops in the network and tends towards its optimal value. The fitness function has various parameters like throughput, packet loss, energy consumed etc. 


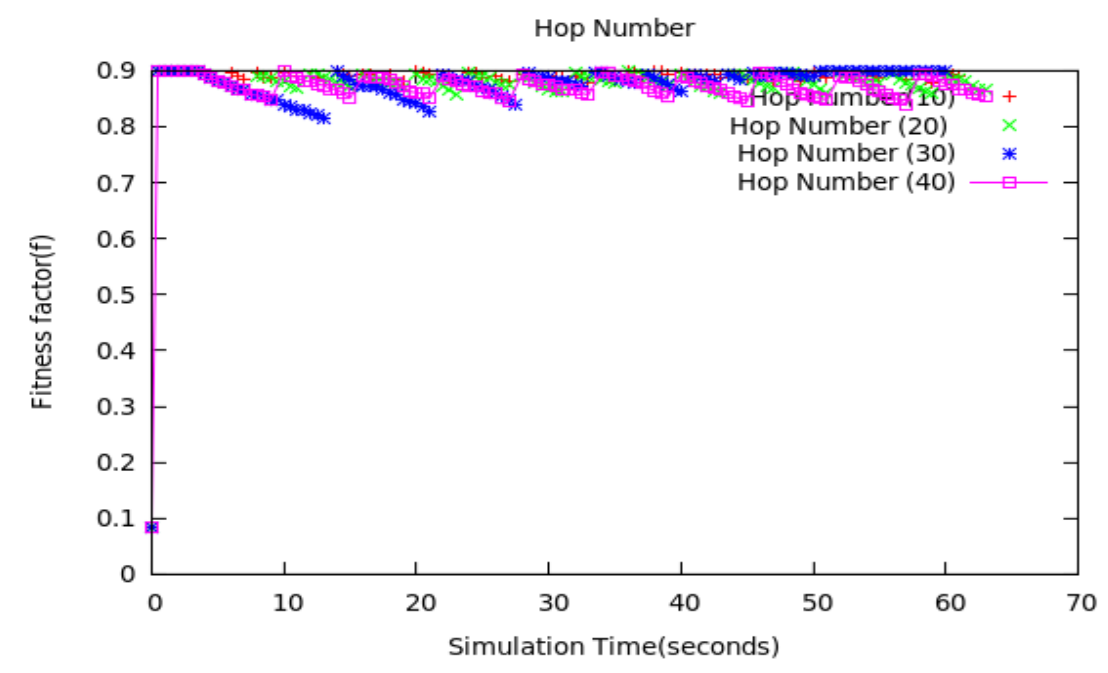

Figure.4 Graph between Simulation Time and Fitness Factor

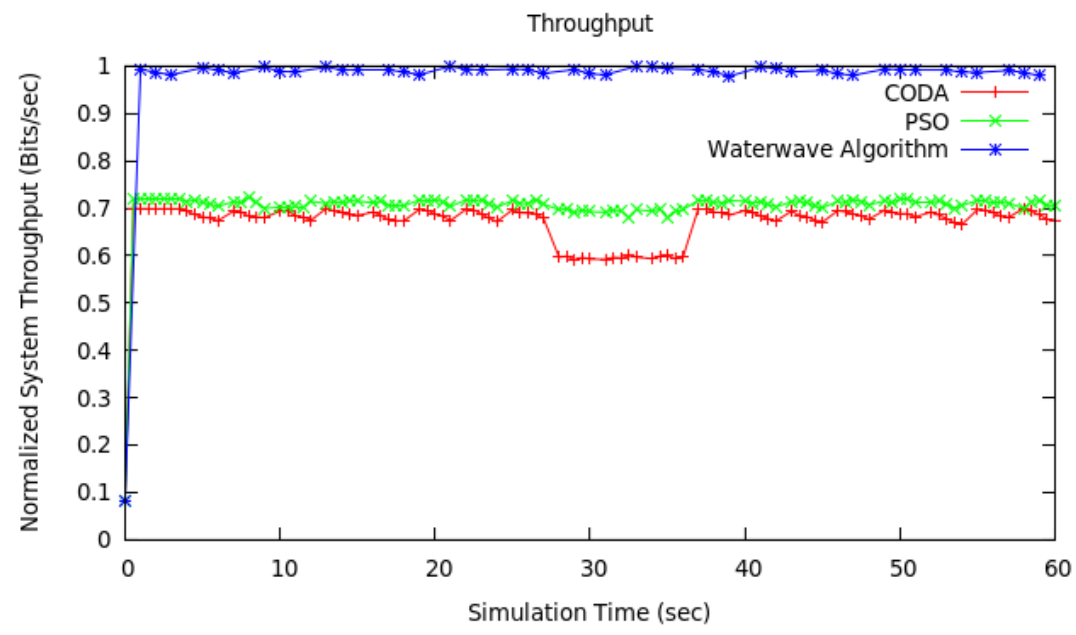

Figure. 5 Throughput of the Network

Figure 5 displays the graph between simulation time and network throughput. The graph compares CODA, PSO and the proposed approach. Water Wave algorithm outperforms the other two algorithms on throughput. Figure 6 displays the graph between the Simulation Time and the Network Lifetime of the nodes in the network. Network Lifetime of nodes in case of water wave optimization algorithm is greater than CODA and PSO because energy consumption of water wave optimization algorithm is low as compared to other algorithms. 


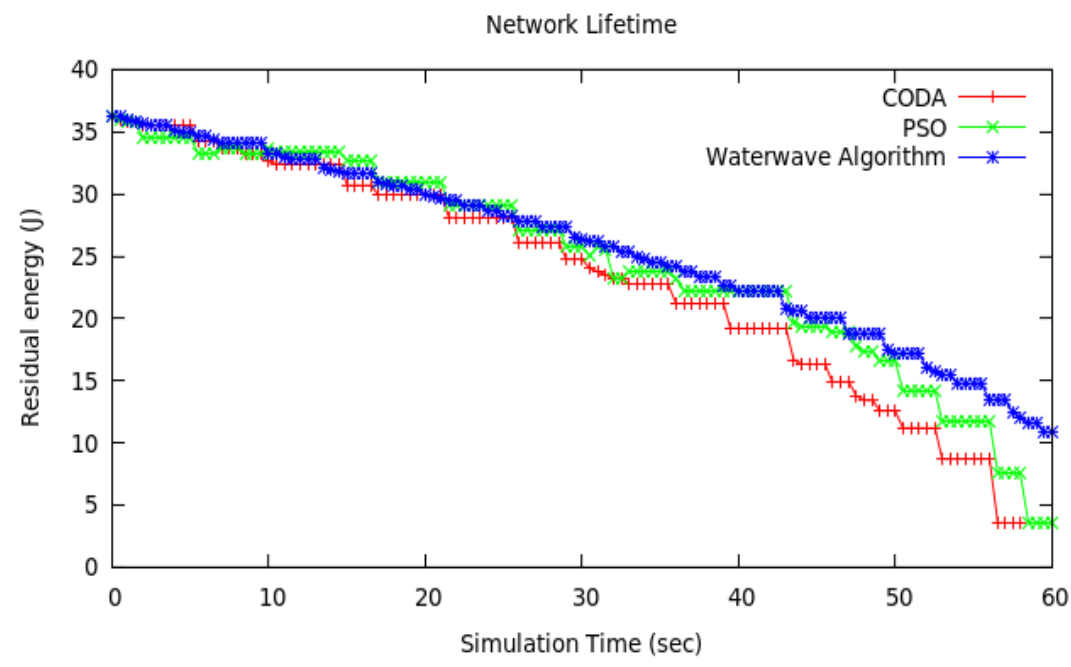

Figure.6 Network Lifetime of Nodes in Network

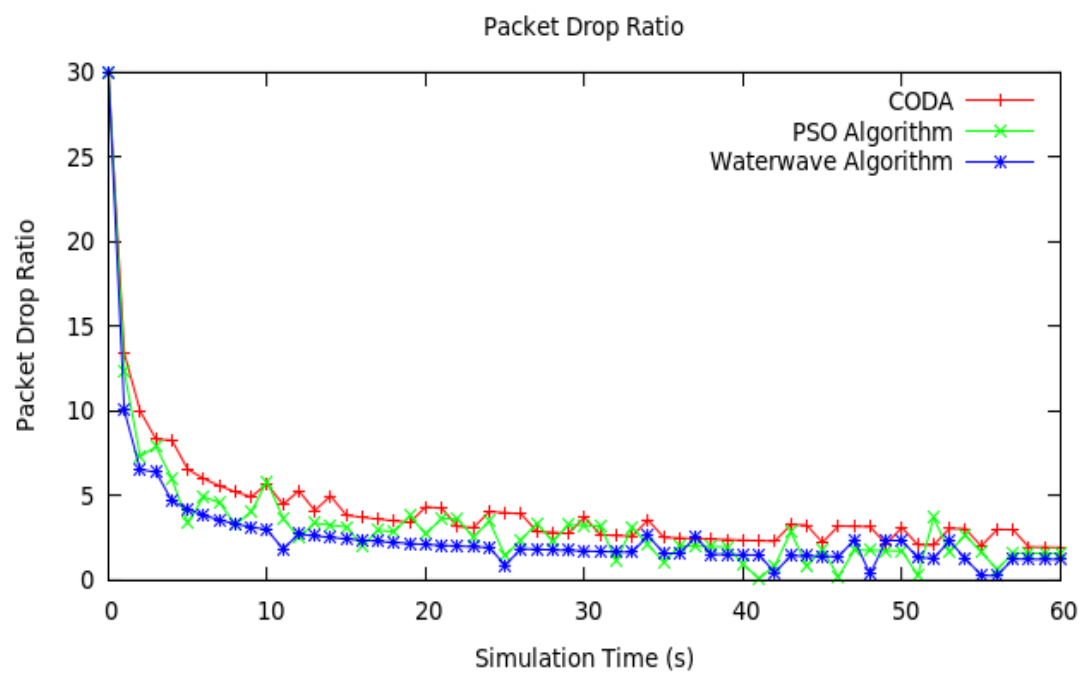

Figure. 7 Packet Drop Ratio of Nodes in Network

Figure 7 shows the packet drop ratio in the network. The water wave optimization algorithm is better as compared to the CODA algorithm and PSO algorithm is performing similar to the water wave algorithm in terms of packets drop ratio. The graphs are designed with hop count 40.

\section{Conclusion and Future Scope}

The results have proved that after implementation of water wave optimization algorithm, Queue length of each node decreases by increasing the number of hops. This proves that the traffic in the network is distributed among all the nodes in the network rather than concentrating on fewer ones. The network lifetime of nodes in the network is higher as compared to the CODA and PSO. Since the residual energy of each node in case of Water-wave algorithm is higher than CODA and PSO. Throughput of the network is also higher in case of Water-wave algorithm and is almost constant around 1. Packet drop ratio is higher in CODA and remains almost same in case of PSO algorithm as compared to the water wave optimization algorithm. Thus water wave optimization algorithm outperforms all other algorithms discussed in this 
Mukhdeep Manshahia; Water Wave Optimization Algorithm based Congestion Control and Quality of Service Improvement in Wireless Sensor Networks, Transactions on Networks and Communications, Volume 5 No. 4 , August (2017); pp: 31-39

work on quality of service parameters. In future other computational intelligence techniques will be implemented and compared with the proposed approach.

\section{REFERENCES}

[1] Kandris, D., Tsioumas, P.; Tzes, A., Nikolakopoulos, G., \& Vergados, D. (2009). Power conservation through energy efficient routing in wireless sensor networks. Sensors, 9, 7320-7342.

[2] Jin, Z., Ping, Y., Wang, Z., Ping, L., \& Guang, L. (2009). A survey on position-based routing algorithms in wireless sensor networks. Algorithms, 2, 158-182.

[3] Alam Bhuiyan and et al. , Energy and bandwidth-efficient wireless sensor networks for monitoring highfrequency events, in: Proceeding of 10th Annual IEEE Communications Society Conference on.Sensor, Mesh and Ad Hoc Communications and Networks (SECON), IEEE, 2013, pp. 194 - 202.

[4] Yick, J., Mukherjee, B., \& Ghosal, D. (2008). Wireless sensor network survey, Computer Networks, 52(12), 2292-2330.

[5] Rawat, P., Kamal Deep Singh, Hakima C., \& Jean Marie Bonnin. (2014). Wireless sensor networks: a survey on recent developments and potential synergies. The Journal of Supercomputing, 68(1), 1-48.

[6] Lo, Shou-Chih, Jhih-Siao Gao, \& Chih-Cheng Tseng. (2003). A water-wave broadcast scheme for emergency messages in VANET. Wireless personal communications, 71(1), 217-241.

[7] I.F. Akyildiz, W. Su, ,Y. Sankarasubramaniam, \& E. Cayirci. (2002). Wireless sensor networks: a survey. Computer Networks, 38(4), 393-422.

[8] Jennifer Yick, Biswanath Mukherjee, \& Dipak Ghosal. (2008), Wireless sensor network survey, Computer Networks, 52(12), 2292-2330.

[9] Stefanos A. Nikolidakis, Dionisis Kandris, Dimitrios D. Vergados, \& Christos Douligeris. (2013). Energy Efficient Routing in Wireless Sensor Networks Through Balanced Clustering. Algorithms, 6, 29-42.

[10] S. Motdhare. (2015). Congestion Control in Wireless Sensor Networks: Mobile Sink Approach. International Journal of Science and Research, 4(1), 2561-2565.

[11] P. Antoniou, A. Pitsillides, T. Blackwell, A. Engelbrecht, \& L. Michael. (2011). Congestion Control in Wireless Sensor Networks based on Bird Flocking Behavior. Computer Networks, 57(5), 1167-1191.

[12] A.K. Luha, T. Vengattraman, \& M. Sathya. (2014). RAHTAP Algorithm for Congestion Control in Wireless Sensor Network. International Journal of Advanced Research in Computer and Communication Engineering, 3(4), 6250-6255.

[13] X. Wei-qiang, \& W. Tie-jun. (2006). Optimal congestion control algorithm for ad hoc networks: Penalty function-based approach. Journal of Zhejiang university SCIENCE A, 7(12), 2110-2117. 
[14] V. Raghunathan, \& P.R. Kumar. (2007). Counterexample in Congestion Control of Wireless Networks. Performance Evaluation, 64(5), 399-418.

[15] C. Zhao, Y. Luo, F. Chen, J. Zhang, \& R. Wang. (2014). Energy Effective Congestion Control for Multicast with Network Coding in Wireless Ad Hoc Network. Mathematical Problems in Engineering, 2014.

[16] Manshahia, M.S., Dave, M. and Singh, S.B., Bio Inspired Congestion Control Mechanism for Wireless Sensor Networks, in: Proceedings of 2015 IEEE International Conference on Computational Intelligence and Computing Research (ICCIC), Madurai, India, December, 2015.

[17] Manshahia, M.S., Dave, M., \& Singh, S.B. (2015). Congestion Control in Wireless Sensor Networks Based on Bioluminescent Firefly Behavior. Wireless Sensor Networks, 7, 149-156.

[18] Yu-Jun Zheng. (2015). Water wave optimization: A New Nature-inspired Metaheuristic. Computers \& Operations Research, 55, 1-11.

[19] Wan. C.Y, Eisenman. S. B., \& Campbell. A. T, CODA: Congestion Detection and Avoidance in Sensor Networks, in: Proceedings of the 1st international conference on Embedded networked sensor systems, SenSys '03, Los Angeles, 2003, pp.266 - 279.

[20] Antoniou P., Pitsillides A., Blackwell T., Engelbrecht A., \& Michael L. (2013). Congestion Control in Wireless Sensor Networks based on Bird Flocking Behaviour Congestion. Computer Networks, 57(5), 1167-1191.

[21] R. C. Eberhart, J. A Kennedy, New optimizer using particle swarm theory, in: Proceedings of the Sixth International Symposium on Micro machine and Human Science, Nagoya, Japan, 1995, pp: 39-43.

[22] Kennedy, J., \& Eberhart, R.C. (1995). Particle swarm optimization, in: Proceedings of IEEE International Conference on Neural Networks, Piscataway, NJ, 1995, pp. 1942-1948.

[23] Manshahia, M.S., Dave, M. and Singh, S.B., Firefly algorithm based clustering technique for Wireless Sensor Networks, in: Proceedings of International Conference on Wireless Communications, Signal Processing and Networking (WiSPNET), 23-25 March 2016.

[24] Akkaya K. \& Younis M. (2005). A Survey of Routing Protocols in Wireless Sensor Networks. Ad Hoc Networks, 3(3), 325-349.

[25] Manshahia, M.S., Dave, M., \& Singh, S.B.(2016). Improved Bat Algorithm Based Energy Efficient Congestion Control Scheme for Wireless Sensor Networks. Wireless Sensor Network, 8, 229- 241. 